\title{
XPS Studies of $\mathrm{SiO}_{2} / \mathrm{Si}$ System under External Bias
}

\author{
Burak Ulgut and Sefik Suzer* \\ Department of Chemistry, Bilkent University, 06533 Ankara, Turkey
}

Received: September 5, 2002; In Final Form: February 6, 2003

\begin{abstract}
Thermally grown $\mathrm{SiO}_{2}$ layers on $\mathrm{Si}(100)$ substrate have been subjected to different external voltage bias during XPS analysis to induce changes in the measured binding energy difference between $\mathrm{Si}^{4+}$ and $\mathrm{Si}^{0}$ in $\mathrm{Si} 2 \mathrm{p}$ and $\mathrm{Si}_{\mathrm{KLL}}$ regions. The $\mathrm{Si} 2 \mathrm{p}$ binding energy difference increases from 3.2 to 4.8 for samples containing 1-7 $\mathrm{nm}$ oxide thickness, and furthermore, this difference can be influenced by application of an external bias to the sample. Application of negative d.c. bias increases the binding energy difference, whereas positive bias decreases it. The voltage dependence of the binding energy difference exhibits a sigmoid character with an abrupt change near $0 \mathrm{~V}$. Both the binding energy difference and differential change between the positive and negative bias have similar functional dependence on the thickness. This is attributed to differential charging between the silicon oxide layer and silicon substrate, which is decreased when a positive bias is applied to the sample (and therefore attracting a larger proportion of the stray electrons from the vacuum chamber to partially neutralize the oxide). Similarly, when negative bias is applied, the stray electrons are repelled from the sample resulting in less neutralization and an increased differential charging. Through external biasing, it is determined that charging in the $\mathrm{SiO}_{2} / \mathrm{Si}$ system persists all of the way down to $1 \mathrm{~nm}$. Application of a.c. (square-wave) bias is equivalent to simultaneous application of negative and positive bias together. However, the differential change in the binding energy difference in the positive and negative cycle is frequency dependent and approaches to the d.c. results at lower frequencies.
\end{abstract}

\section{Introduction}

The $\mathrm{SiO}_{2} / \mathrm{Si}$ system has been extensively studied using various spectroscopic techniques. XPS, X-ray photoelectron spectroscopy, is one of the most popular because of its capability to differentiate among the various chemical states by means of experimentally determined chemical shifts. In the $\mathrm{SiO}_{2} / \mathrm{Si}$ system, the measured chemical shift between $\mathrm{Si}^{4+}$ and $\mathrm{Si}^{0}$ has been reported to increase (from ca. 3.2 to $5.0 \mathrm{eV}$ ) as a function of the oxide thickness $(1-10 \mathrm{~nm})$. This increase has been the subject of a long debate and is attributed to various chemical and/or physical parameters. ${ }^{1-11}$ In general, three components contribute to the measured chemical shifts in XPS analysis. They are (i) initial-state effects which are mostly chemical, (ii) finalstate effects (extra-atomic relaxation) which are mostly physical (environment dependent), and (iii) charging which is extrinsic and instrument dependent. In a review article, Iwata and Ishizaka ${ }^{12}$ compiled and discussed these different components. They concluded that for the $\mathrm{SiO}_{2} / \mathrm{Si}$ system with oxide thickness of 1,3 , and $4 \mathrm{~nm}$ the true chemical shift is $3.0 \pm 0.2 \mathrm{eV}$, and the difference from the measured chemical shift $(3.5-4.5 \mathrm{eV})$ stems mostly from charging induced by the photoemission process. Zhang et al., ${ }^{13,14}$ constructing structurally homogeneous silicon oxide films consisting of $\mathrm{H}_{8} \mathrm{Si}_{8} \mathrm{O}_{12}$ or $\mathrm{H}_{10} \mathrm{Si}_{10} \mathrm{O}_{15}$ layers on silicon, claimed to have minimized the initial state effects and directly measured magnitude of the final state effects. From the analysis of their data, they concluded that the onset of the charging was $3 \mathrm{~nm}$. In a recent article, Kobayashi et al. ${ }^{15}$ reported that the increase of the energy shift on the oxide thickness almost disappeared with the deposition of a thin

* To whom correspondence should be addressed. E-mail: suzer@ fen.bilkent.edu.tr. palladium layer as a result of the elimination of the surface charging effect.

Charging, most commonly referred to as surface charging, as a result of the photoelectron emission from insulators, has been a serious problem since the early developments of XPS (or ESCA), and various instrumental techniques have been developed to overcome it. ${ }^{16-21}$ The most common technique involves use of a low energy flood gun for charge neutralization. $^{22}$

Constructive use of the surface charging phenomenon for structural/chemical determinations has also been reported. Lau and co-workers ${ }^{23-29}$ have published a number of articles dealing with various aspects of the use of the surface charging for extracting structural and/or electrical properties of ultrathin dielectric films on semiconductors. Thomas et al. ${ }^{30}$ have used charging to separate the surface spectrum (mainly silicon dioxide) from the silicon substrate spectrum (consisting of contamination and silicon dioxide on silicon). Elegant use of surface charging for lateral differentiation of mesoscopic layers and for depth profiling in 1-10 $\mathrm{nm}$ thin layers have recently been reported. ${ }^{31,32}$ In most of these studies, surface charging was controlled/varied via a low energy electron flood gun. In a recent report, Havercroft and Sherwood ${ }^{33}$ demonstrated that simple external biasing of the sample holder by a d.c. power supply can also be used to induce differential surface charging. They showed that sample biasing using a large negative d.c. voltage $(25-100 \mathrm{~V})$ could be used to identify chemical differences in oxide films on an aluminum alloy. The present work reports similar but low $(1-20 \mathrm{~V})$ d.c. (both positive and negative) or a.c. (square-wave) external biasing to investigate the charging issue in the $\mathrm{SiO}_{2} / \mathrm{Si}$ system without and with a thin layer of gold deposited. 

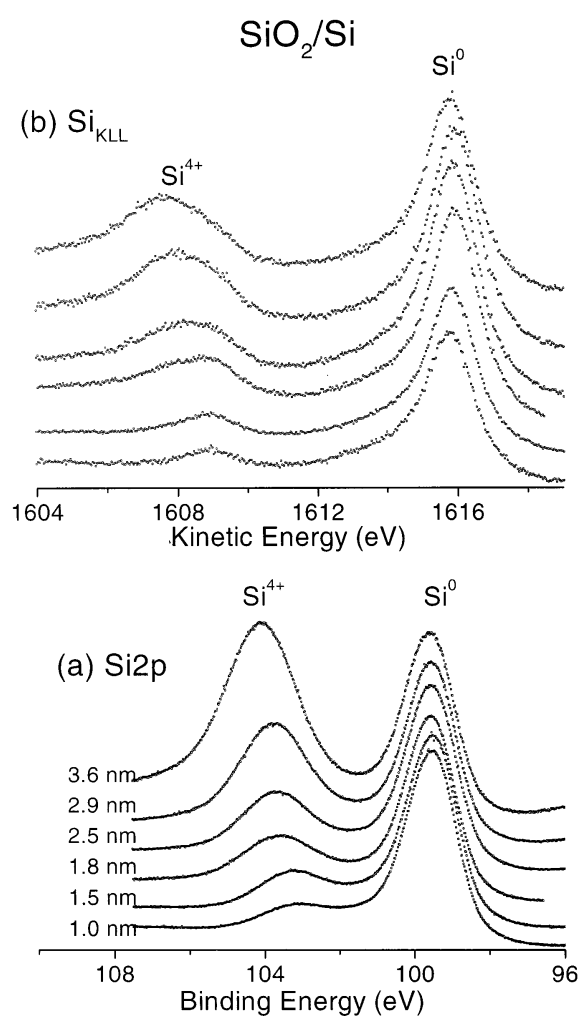

Figure 1. XPS spectra (using non monochromatic $\mathrm{MgK} \alpha$ source) of 6 different thermally grown silicon dioxide layers on silicon substrate: (a) $\mathrm{Si} 2 \mathrm{p}$ region and (b) $\mathrm{Si}_{\mathrm{KLL}}$ region.

\section{Experimental Section}

Oxide layers were grown thermally on HF-cleaned $\mathrm{Si}$ (100) substrates at $500{ }^{\circ} \mathrm{C}$ in air for various duration. Angle-resolved XPS is used to estimate the thickness of the overlayer. ${ }^{34}$ In some measurements, the $\mathrm{SiO}_{2} / \mathrm{Si}$ system is used for deposition of thin layers of gold via (i) direct deposition from $0.001 \mathrm{M}$ aqueous $\mathrm{HAuCl}_{4}$ solution or (ii) vapor deposition by sputtering from a gold metal target in a vacuum. A Kratos ES300 electron spectrometer with $\mathrm{MgK} \alpha \mathrm{X}$-rays (nonmonochromatic) is used for XPS analysis. A typical sample is a ca. 1-mm-thick silicon wafer with dimensions of $4 \times 12 \mathrm{~mm}$. In the standard geometry, the sample accepts $\mathrm{X}$-rays at $45^{\circ}$ and emits photoelectrons at $90^{\circ}$ with respect to its surface plane. The sample can also be rotated to decrease the emission angle (electron takeoff angle) in order to enhance surface sensitivity (keeping the X-raysample-analyzer angle always at $45^{\circ}$ ). The $\mathrm{X}$-ray power was varied from 50 to $300 \mathrm{~W}$ to check the flux dependence. Samples were electrically connected both from the top (oxide layer) and the bottom (silicon substrate) to the sample holder, which was grounded or biased with a d.c. (or a.c.) power supply externally. Additional measurements were also carried out where the sample was connected from the bottom-only in order to assess the effects of different connecting/grounding schemes.

\section{Results}

Figure 1 depicts $\mathrm{Si} 2 \mathrm{p}$ and $\mathrm{Si}_{\mathrm{KLL}}$ Auger peaks corresponding to different samples, clearly indicating that the chemical shift between $\mathrm{Si}^{4+}$ and $\mathrm{Si}^{0}$ peak increases as a function of the oxide thickness as has been reported numerous times in the literature. ${ }^{1-15}$ Because the unmonochromatized X-ray anodes also emit a substantial amount of continuous energy X-rays (Bremstrahlung), Auger peaks are also abundant in a typical XPS spectrum. For the $\mathrm{SiO}_{2} / \mathrm{Si}$ system, the kinetic energy of the corresponding $\mathrm{Si}_{\mathrm{KLL}}$ peaks are around $1610 \mathrm{eV}$ which fall outside the binding energy

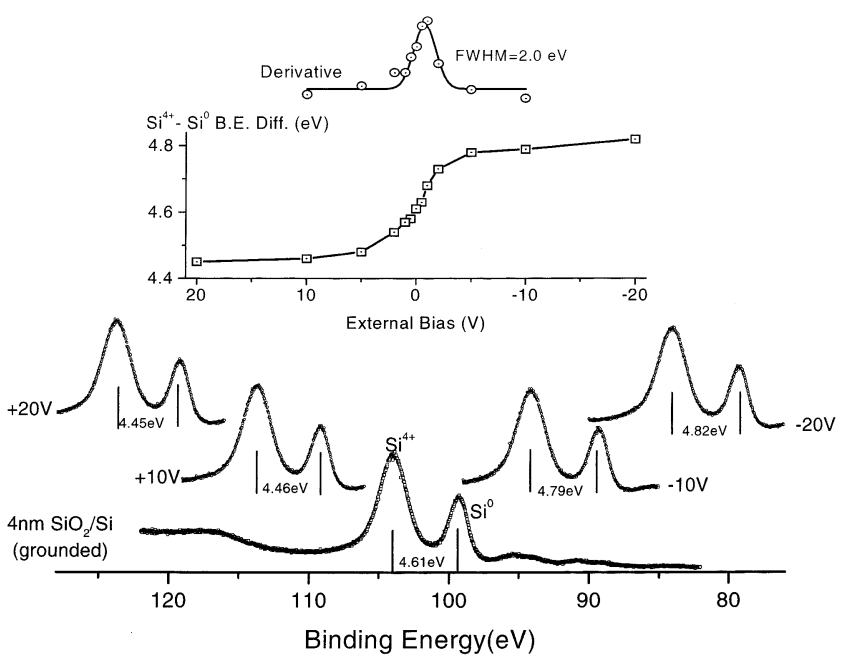

Figure 2. XPS spectra of $4 \mathrm{~nm} \mathrm{SiO}_{2} / \mathrm{Si}$ system recorded without and with external d.c. bias. The inset shows the Si2p binding energy difference between the oxide $\left(\mathrm{Si}^{4+}\right)$ and the substrate $\left(\mathrm{Si}^{0}\right)$ as a function of the applied bias together with its first derivative.

range used for the $\mathrm{Mg} \mathrm{K} \alpha$ source $(0-1250 \mathrm{eV})$, hence one has to scan the negative binding energy region $(-360 \mathrm{eV})$ to record them. The chemical shift in the Auger region is approximately 2 times larger than that in the Si2p region which can be roughly attributed to the fact that 2 holes are involved in the Auger process as opposed to one hole in the photoemission process..$^{35}$

A. d.c. Biasing. External biasing with a negative d.c. source shifts the absolute position of the peaks to lower binding energies (increasing their kinetic energy) and a positive d.c. source to higher energies as shown in Figure 2. More importantly, the chemical shift difference between the $\mathrm{Si}^{4+}$ and the $\mathrm{Si}^{0}$ peaks increases under negative bias and decreases under positive bias as also shown in the same figure. This difference under bias, as shown in the insert, displays a sigmoid character around $0 \mathrm{~V}$, and within $2 \mathrm{~V}$, it reaches saturation at both ends. The first derivative of the binding energy difference, which is also displayed in the figure, is a Gaussian-like curve with fwhm of $2.0 \mathrm{eV}$.

In Figure 3, we display the dependence of the binding energy difference on the thickness of the $\mathrm{SiO}_{2}$ overlayer without and with -10 and $+10 \mathrm{~V}$ external bias. The difference between negative and positive bias is also plotted in the same figure, indicating that the externally induced shift, which will be claimed to be due to differential charging later in the discussion section, displays a very similar behavior with respect to the thickness of the oxide layer and, more importantly, persists down to $1 \mathrm{~nm}$. Accordingly, while the binding energy difference between $\mathrm{Si}^{4+}$ and the $\mathrm{Si}^{0}$ peaks increases from 3.2 to $4.8 \mathrm{eV}$ when grounded, the bias induced difference between the negative and positive $10 \mathrm{~V}$ similarly increases from 0 to ca. 0.30 $\mathrm{eV}$. Furthermore, the bias-induced shifts for the Si2p and the $\mathrm{Si}_{\mathrm{KLL}}$ peaks are numerically equal to each other within our experimental errors. Taking into account that our experimental error in determining the binding energy difference to be better than $0.03 \mathrm{eV}$, the measured bias-induced shifts are considerable. ${ }^{36}$ It is also interesting to note that Au deposited on the $\mathrm{SiO}_{2}$ overlayer follows exactly the same changes as the overlayer itself and not as the silicon substrate (Figure 4).

The bias-induced shifts were observable both when the samples were connected from the bottom and top together or from the bottom-only (by silver paint).

B. a.c. Biasing. Application of a square wave (with different frequencies) is equivalent to application of both negative 

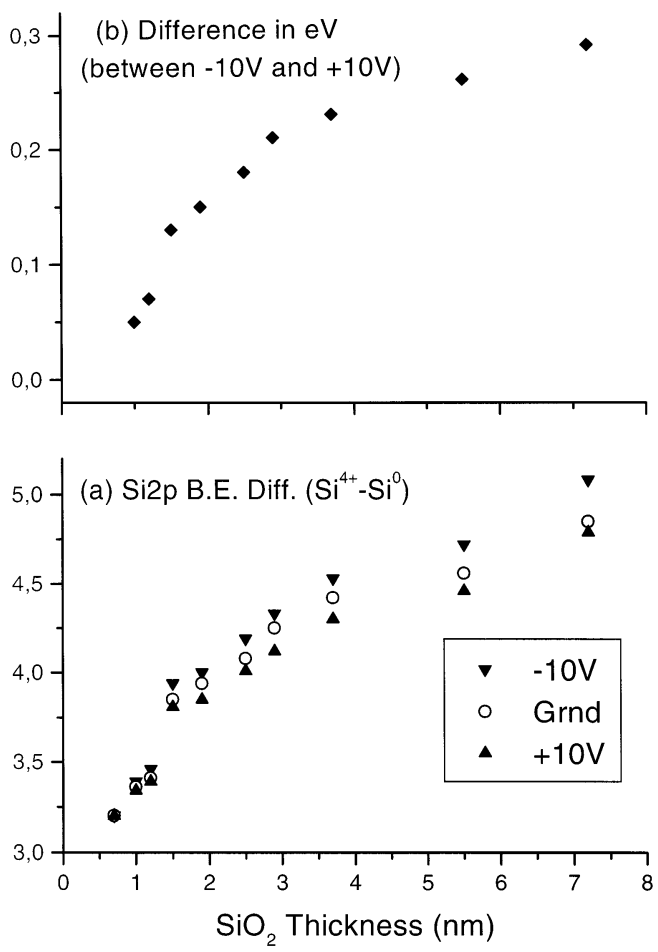

Figure 3. (a)Variation of the Si2p binding energy difference between the oxide $\left(\mathrm{Si}^{4+}\right)$ and the substrate $\left(\mathrm{Si}^{0}\right)$ with the thickness of the oxide layer recorded without and with application of +10 and $-10 \mathrm{~V}$ bias. (b) Variation of the binding energy difference between the sample subjected to -10 and $+10 \mathrm{~V}$ bias with the thickness of the oxide layer.

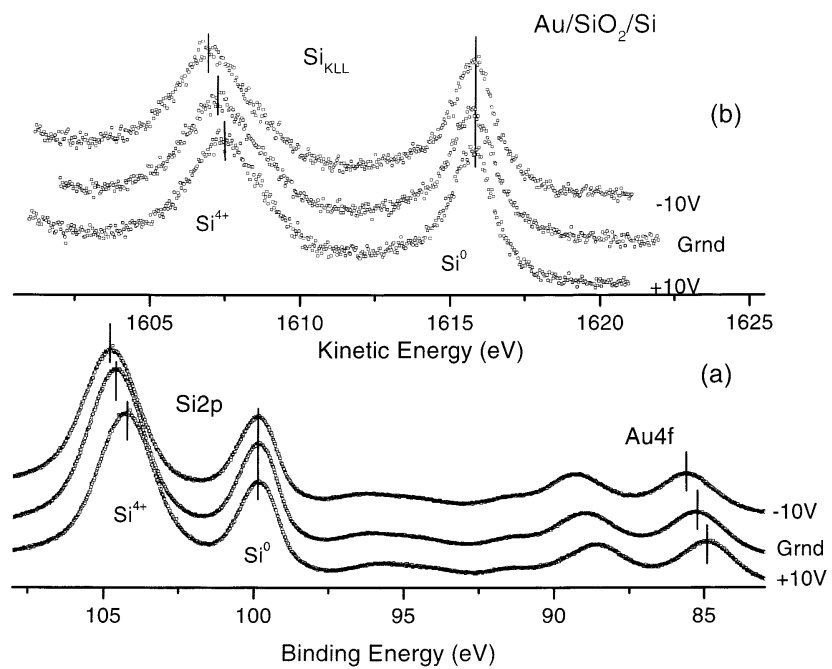

Figure 4. XPS spectra of $4 \mathrm{~nm} \mathrm{SiO}_{2} / \mathrm{Si}$ with gold deposited on the overlayer without and with -10 and $+10 \mathrm{~V}$ external bias: (a) Si2p$\mathrm{Au} 4 \mathrm{f}$ region and (b) $\mathrm{Si}_{\mathrm{KLL}}$ region.

and positive bias simultaneously as shown in Figure 5. Here again, the energy difference between $\mathrm{Si}^{4+}$ and $\mathrm{Si}^{0}$ varies and becomes larger. For high frequencies, the binding energy differences at the negative cycle and the positive one are similar, but at sufficiently low frequencies (approaching d.c.), the binding energy difference becomes lower and higher at positive and negative cycles, respectively, as shown in the Figure 5 revealing an additional frequency dependent (dynamic) component.

C. X-ray Flux Dependence. In all of the experiments we performed, we could not detect any significant dependence on the flux of the X-rays (between 50 and $300 \mathrm{~W}$ ) whether grounded or under external bias.

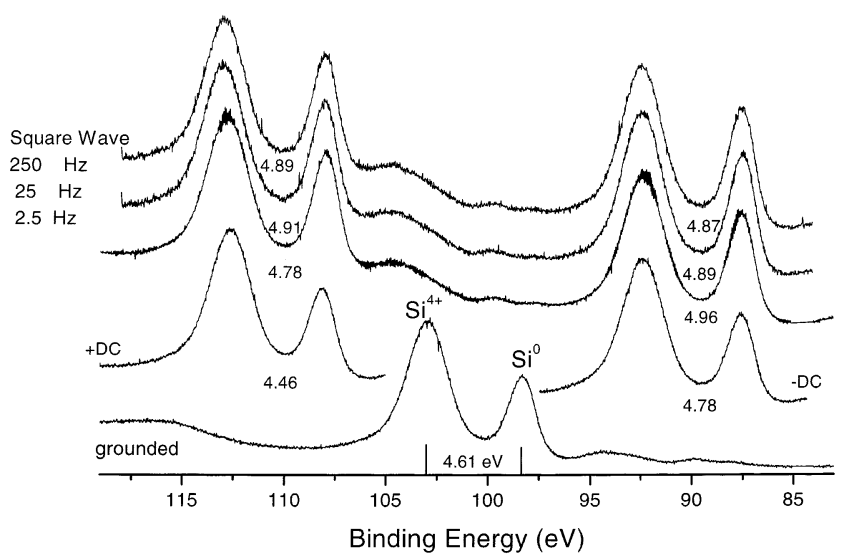

Figure 5. XPS spectra of $4 \mathrm{~nm} \mathrm{SiO}_{2} / \mathrm{Si}$ system recorded without and with external d.c. bias together with a.c. (square-wave) bias at 3 different frequencies.

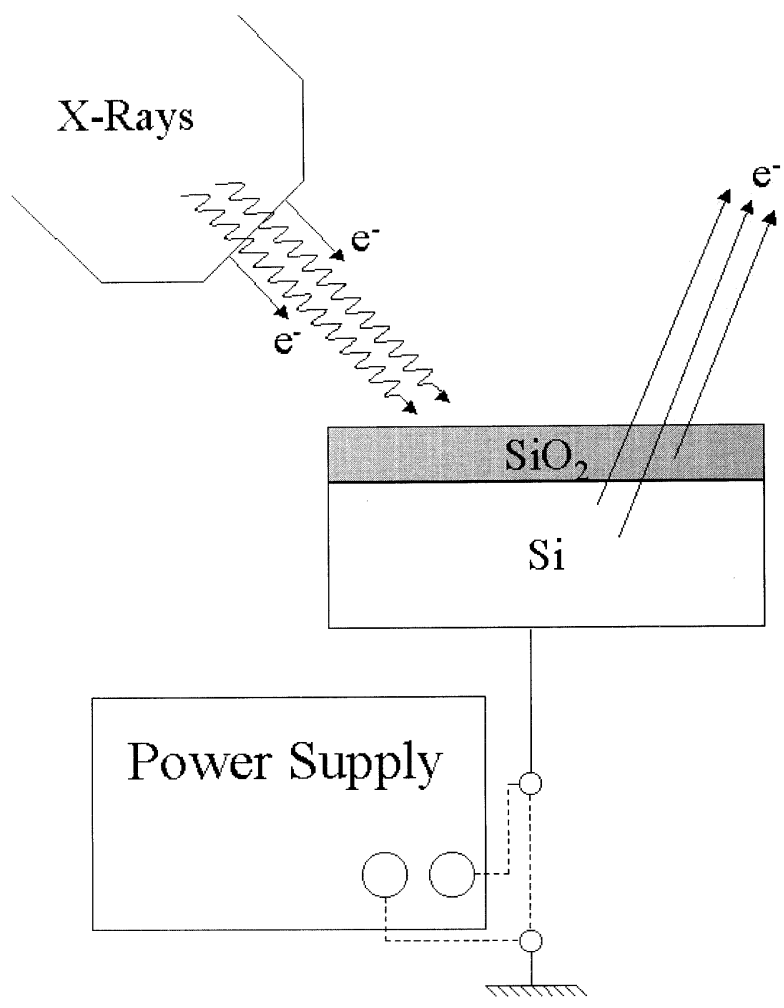

Figure 6. Schematic diagram of the XPS setup with external bias.

\section{Discussion and Conclusions}

During photoemission, electrons are emitted from the sample and these electrons are replenished via the ground connection. However, for samples with low electrical conductivity, a net surface positive charge develops because of a difference between the rate of photoemission and rate of replenishment of the electrons as illustrated in Figure 6. This has been given as the reason for the increase of the chemical shift between the silicon oxide layer and the silicon substrate. ${ }^{1-12}$ However, some of the surface charging may be neutralized by stray electrons within the vacuum chamber originating from the X-ray source and/or ion gauge(s). When the sample is subjected to external negative bias, the stray electrons are repelled from the sample, causing an even larger charging and a larger binding energy difference between the oxide and the substrate. When positive bias is applied, a larger proportion of the stray electrons are attracted toward the sample holder causing a larger proportion of neutralization (hence a smaller binding energy difference). In 

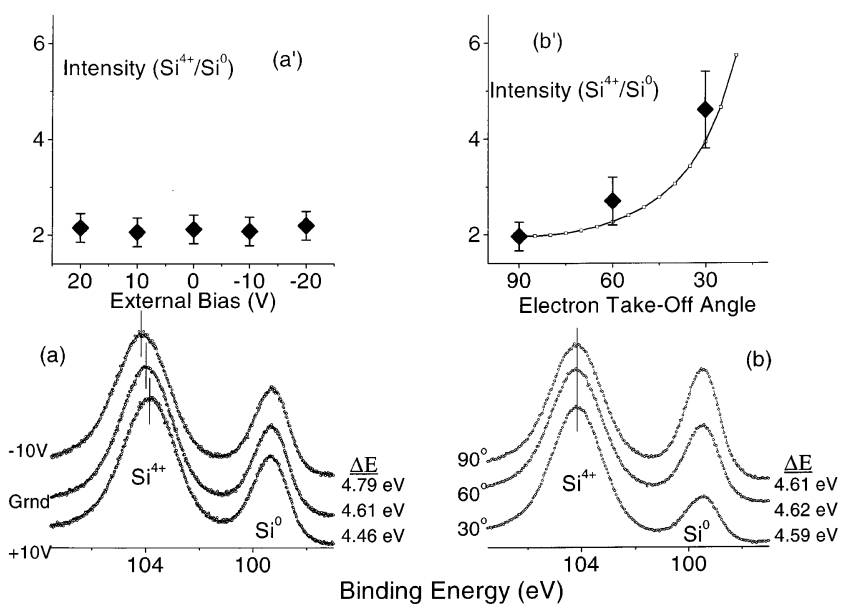

Figure 7. XPS spectra of $4 \mathrm{~nm} \mathrm{SiO}_{2} / \mathrm{Si}$ system: (a) recorded without and with -10 and $+10 \mathrm{~V}$ bias under $90^{\circ}$ electron takeoff angle, (a') the Si2p intensity ratio of the oxide $\left(\mathrm{Si}^{4+}\right)$ to the substrate $\left(\mathrm{Si}^{0}\right),(\mathrm{b})$ recorded at different electron takeoff angles, $\left(b^{\prime}\right)$ the intensity ratio together with the expected $1 / \sin$ (electron takeoff angle). ${ }^{22}$

this respect, the stray electrons within the vacuum system act as a simple and cheap low energy electron flood gun. The sigmoid character of the binding energy difference around $0 \mathrm{~V}$ together with the Gaussian-like derivative and $2 \mathrm{~V}$ fwhm (Figure 2) also support this argument.

Another point is that the change of the angular acceptance of the collected electrons with external bias would increase with a negative bias and decrease with a positive one. Accordingly, an increase in the angular acceptance would lead to an increase in the surface sensitivity; hence, the $\mathrm{SiO}_{2}$ components on the surface having a higher binding energy would contribute more leading to an increase in the binding energy difference between the oxide and the substrate. ${ }^{12,22}$ To check the validity of this claim, we have reproduced the intensity ratio of the oxide to substrate Si2p peaks in Figure 7. As depicted in the figure, the intensity ratio stays constant (within the experimental error limits which is believed to be around 10\%) when the sample is subjected to -20 to $+20 \mathrm{~V}$ external bias, although the binding energy difference changes as was already shown in Figure 2. Figure 7 also depicts the intensity ratio when the sample is intentionally rotated to enhance the surface sensitivity. In this case, although the ratio followed the expected $1 / \sin$ (takeoff angle) the binding energy difference stayed the same. ${ }^{22}$ Such an effect was indeed observed (i.e., increase of the oxide/ substrate intensity under negative bias and decrease under positive one) only when very large biases are applied (more than $100 \mathrm{~V}$ ) but is definitely not operative at small biases.

Another source for these neutralizing slow electrons is the secondary electrons generated within the sample upon X-ray exposure. When a negative bias is applied, more electrons are emitted increasing the charging. When a positive bias is applied, the electrons stay within the sample and are not emitted so that charging is reduced. ${ }^{37}$

Whatever the origin of the neutralizing electrons is, the external bias induces small but significant binding energy differences between the silicon oxide layer and the silicon substrate. The reason for our failure to observe any significant X-ray flux dependence may also be related to this fact. As the flux of the $\mathrm{X}$-rays increases, more stray electrons (as well as more secondary electrons) are probably produced to offset the increase in differential charging. Hence, we now claim that detection of a measurable difference under positive and/or negative bias is a direct proof of the presence of differential charging.
Binding energy differences between $\mathrm{Si}^{4+}$ and $\mathrm{Si}^{0}$ for the $\mathrm{Si} 2 \mathrm{p}$ XPS and the $\mathrm{Si}_{\mathrm{LVV}}$ Auger regions were recently calculated based on electrostatic theory in which the extra atomic relaxation (or core screening) was shown to be effective and varied inversely with the distance from the oxide/Si interface. ${ }^{38,39}$ Accordingly, Kobayashi et al. ${ }^{15}$ attributed the Si2p B.E. shift (displaying an inverse oxide-thickness dependence) to mostly extra atomic relaxation in the range of $0-2 \mathrm{~nm}$ and claimed to have determined that the true chemical shift of silicon oxide layer thicker than $2 \mathrm{~nm}$ to be ca. $3.8 \mathrm{eV}$. For samples thicker than 4 $\mathrm{nm}$, they attributed the B.E. difference to charging which was modeled using a linear dependence on the thickness and the capacitance of the oxide layer. For samples having a thickness from 2 to $4 \mathrm{~nm}$, they considered both the screening and charging effects together in agreement with their experimental findings. A similar conclusion was also reached by Zhang et al. ${ }^{13,14}$ where they claimed that $\alpha-\mathrm{SiO}_{2}$ films should not charge until at least 3-4 nm thick under X-ray fluxes similar to those used in their experiment (200-400 W). Our experimental findings for the functional dependence of the B.E. difference on the oxide thickness as shown in Figure 3 are in agreement with these findings. In addition, we show that charging (under similar X-ray fluxes), as revealed by increase or decrease in the B.E. difference after application of negative and positive external voltage bias, respectively, also displays a very similar functional dependence on the thickness of the oxide layer. We also demonstrate that the differential charging is effective all of the way down to 1 $\mathrm{nm}$. This is in sharp contrast with the earlier claims ${ }^{13-15}$ but is in complete agreement with Iwata and Ishizaka. ${ }^{12}$ Our finding is further supported by the fact that when gold is deposited on the oxide the Au4f peaks shift together with the oxide Si2p peak indicating that no differential charging exits between the gold particles and the ultrathin silicon oxide layer, although severe differential and externally variable charging exists between the oxide layer and the silicon substrate.

Hence, a simple external bias can be a very useful diagnostic tool to establish whether differential charging is operative and to investigate the proximity of the surface atoms (i.e., Au on the oxide) in nanometer scale structures. External biasing can also be used to differentiate XPS peaks with respect to their lateral or spatial distributions or electrical properties in heterogeneous samples. One disadvantage is that because the number density of the stray electrons (or the secondary electrons emitted) is not controllable we can not expect to completely eliminate the differential charging using this simple external bias as opposed to use of a flood gun.

As was depicted in Figure 5, there is additional timedependent binding energy differences which may lead to extract further information like capacitance and/or impedance of these nanometer scale samples using a.c. biasing which will be the subject of our future investigation(s).

Acknowledgment. We are grateful to Dr. Cemal Yalabik and Dr. Ahmet Oral of Bilkent Physics Department, Turkey and Dr. Hagai Cohen of the Weizmann Institute, Israel for various suggestions and discussions.

\section{References and Notes}

(1) Clarke, R. A.; Tapping, R. L.; Hopper, M. A.; Young. Y. J. Electrochem. Soc. 1975, 122, 1348.

(2) Hollinger, G.; Jugnet, Y.; Pertosa, P.; Duc, T. M.Chem. Phys. Lett. 1975, 36, 441.

(3) Grunthaner, F. J.; Grunthaner, P. J.; Vasquez, R. P.; Lewis, B. F.; Maserjian, J.; Madhukar, A. Phys. Rev. Lett. 1979, 43, 1683.

(4) Grunthaner, F. J.; Grunthaner, P. J.; Vasquez, R. P.; Lewis, B. F.; Maserjian, J.; Madhukar, A. J. Vac. Sci. Technol. 1979, 16, 1443. 
(5) Ishizaka, A.; Iwata, S.; Kamigaki, Y. Surf. Sci. 1979, 84, 355.

(6) Hollinger, G. Appl. Surf. Sci. 1981, 8, 318.

(7) Hollinger, G.; Himpsel, F. J. Appl. Phys. Lett. 1983, 44, 93.

(8) Wagner, C. D.; Joshi, A.; Gulbrandsen, L.; Deal, B. E. J. Vac. Sci. Technol. B 1984, 2, 107.

(9) Finster, J.; Schulze, D.; Bechstedt, F.; Meisel, A. Surf. Sci. 1985, 152/153, 1063.

(10) Iqbal, A.; Bates, C. W., Jr.; Allen, J. W. Appl. Phys. Lett. 1985, 47,93

(11) Grunthaner, F. J.; Grunthaner, P. J. Mater. Sci. Rep. 1986, 1, 65.

(12) Iwata, S.; Ishizaka, A. J. Appl. Phys. 1996, 79, 6653.

(13) Zhang, K. Z.; Greeley, J. N.; Banaszak Holl, M. M.; McFeely, F.

R. J. Appl. Phys. 1997, 82, 2298.

(14) Zhang, K. Z.; Banaszak Holl, M. M.; McFeely, F. R. J. Phys. Chem. $B$ 1998, 102, 3930 .

(15) Kobayashi, H.; Kubota, T.; Kawa, H.; Nakato, Y.; Nishiyama, N. Appl. Phys. Lett. 1998, 73, 933.

(16) Johansson, G.; Hedman, J.; Berndtsson, A.; Klasson, M.; Nilsson,

R. J. Electron. Spectrosc. Relat. Phenom. 1973, 2, 295.

(17) Dickinson, T.; Povey, A. F.; Sherwood, P. M. A. J. Electron. Spectrosc. Relat. Phenom. 1973, 2, 441.

(18) Lewis, R. T.; Kelly, M. A. J. Electron. Spectrosc. Relat. Phenom. 1980, 20, 105

(19) Barr, T. L. J. Vac. Sci. Technol. A 1989, 7, 1677.

(20) Cros, A. J. Electron. Spectrosc. Relat. Phenom. 1992, 59, 1.

(21) Cazaux, J. J. Electron. Spectrosc. Relat. Phenom. 1999, 105, 155.

(22) Seah, M. P. In Practical Surface Analysis, 2nd ed.; Briggs, D.

Seah, M. P., Eds.; Wiley: New York, 1999; Vol. 1, p 541.

(23) Lau, W. M. J. Appl. Phys. 1989, 65, 2047.

(24) Lau, W. M. Appl. Phys. Lett. 1989, 54, 338

(25) Lau, W. M. J. Appl. Phys. 1990, 67, 1504.
(26) Lau, W. M.; Jin, S.; Wu, X. W.; Ingrey, S. J. Vac. Sci. Technol. B 1990, 8,848

(27) Lau, W. M.; Wu, X. W. Surf. Sci. 1991, 245, 345.

(28) Chan, R. W. M.; Kwok, R. W. M.; Lau, W. M. J. Appl. Phys. 1996, 79,3635 .

(29) Chan, R. W. M.; Kwok, R. W. M.; Lau, W. M.; Yan, H.; Wong,

S. P. J. Vac. Sci. Technol. A 1997, 15, 2787.

(30) Thomas, J. H., III.; Bryson, C. E., III.; Pampalone, T. R. J. Vac. Sci. Technol. B 1988, 6, 1097.

(31) Shabtai, K.; Rubinstein, I.; Cohen, S. R.; Cohen, H. J. Am. Chem. Soc. 2000, 122, 4959 .

(32) Doron-Mor, I.; Hatzor, A.; Vaskevich, A.; van der Boom-Moav, T.; Shanzer, A.; Rubinstein, I.; Cohen, H. Nature 2000, 406, 382.

(33) Havercroft, N. J.; Sherwood, P. M. A. Surf. Interface Anal. 2000 29,232

(34) Mitchell, D. F.; Clark, K. B.; Bardwell, J. A.; Lennard, W. N.; Massoumi, G. R.; Mitchell, I. V. Surf. Interface Anal. 1994, 21, 44.

(35) Wagner, C. D. Anal. Chem. 1972, 44, 972.

(36) Resolution of our spectrometer is slightly better than $0.80 \mathrm{eV}$ as measured in the Ag3d peaks, and we use standard curve fitting routines with $0.60 \mathrm{eV}$ spin-orbit parameter for the Si2p. Because we extract the binding energy difference by fitting the entire silicon substrate and the oxide peaks, we estimate our error in measuring the binding energy differences to be better than $0.03 \mathrm{eV}$.

(37) This point was suggested by Dr. H. Cohen, as well as by one of the reviewers and will be the subject of our future investigations.

(38) Browning, R.; Sobolewski, M. A.; Helms, C. R. Phys. Rev. B 1988, $38,13407$.

(39) Pasquarello, A.; Hybertson, M. S.; Car, R. Phys. Rev. B 1996, 53, 10942. 